\title{
Early Intervention Mechanism for Preventing Electrical Shocks During Construction Projects: Portable Electrical Equipment
}

\author{
Yau-Ren Shiau ${ }^{1}$, Fang-Yi $\mathrm{Lo}^{2}$, and Po-Cheng $\mathrm{Ko}^{3 *}$ \\ ${ }^{1}$ Department of Industrial Engineering and Systems Management, Feng Chia University, 40724 Taichung, Taiwan \\ ${ }^{2}$ Department of International Business, Feng Chia University, 40724 Taichung, Taiwan. \\ ${ }^{3}$ Ph.D. Program of Business, Feng Chia University, 40724 Taichung, Taiwan.
}

\begin{abstract}
To prevent electrical shock accidents in construction sites, the present researchers used portable electrical equipment as an example to plan a preconstruction early intervention mechanism that can be used to conduct various inspections of portable electrical devices. This study used narrative text analysis for data collection and compilation. The researchers analyzed 41 real electrocution death cases involving portable electric equipment as the electrocution medium in the Taiwanese construction industry and identified hazard factors that cause electrocution from the case summaries. Then, the IDEF3 was used to integrate and construct a model for the portable electrical equipment inspection flowchart of the early intervention mechanism as a safety inspection system to prevent electrocution in construction engineering units. This study revealed hazard factors and management omissions related to electrocutions caused by portable electrical equipment. To protect workers and strengthen the safety of the construction site, this study proposed of an electric shock prevention early intervention mechanism for portable electric equipment in construction projects. Various inspections should be conducted before equipment is brought on site for construction operations to ensure the safety of electrical equipment and reduce electrocution risks. This study also established a visualization mechanism for the visual qualification label of portable electrical equipment. This mechanism is conducive to strengthening safety management.
\end{abstract}

\section{Introduction}

Occupational health and safety (OHS) has always been crucial for the construction industry. According to statistics from the 2003-2013 labor force survey conducted by the National Safety Council of the United States [1], although construction workers constituted less than $5 \%$ of the total US labor force, the occupational hazard death toll of construction workers was up to $20 \%$. In a study that investigated of over 60,000 accidents in the US electrical power industry, death by electrocution was the second leading cause of death [2]. According to Taiwan's 2004-2017 construction industry occupational hazard statistics, death by electrocution was the third leading cause of occupational fatality (accumulated death toll: 168 workers) [3]. Electrocution clearly constitutes a great risk factor for casualty accidents at construction sites.

Construction projects typically involve temporary and complex multilayered subcontract operations. Some construction workers may not even be regular members of their construction team and are withdrawn once the construction period is over. Therefore, topics related to safety facilities and management of construction sites have received the attention of various scholars. In the construction domain, safety climate and safety behavior are closely related. Despite the implementation of a

\footnotetext{
* Corresponding author: pochengko1@ gmail.com
}

safety management system, the enforcement of safety plans by high-level management personnel remains insufficient. This causes the casualty rate in the construction industry to remain high [4]. Therefore, drafting, monitoring, and implementing intervention measures for high-risk occupations to reduce severe injuries is an urgent problem [2].

Safety management system techniques are constantly being developed and innovated upon, and many of these techniques detect real-time dangers [5], such as by simulating building safety cyberphysical systems [6], applying radio-frequency identification wireless sensing technology to detect real-time positions, and issuing safety distance alerts at construction sites $[7,8]$. Due to the varied purposes and building operations of construction projects, various types of portable electrical equipment are needed for different construction projects. The results of a US occupational injury survey indicated that welders and power line workers ranked second among occupational injury victims by injury rate; in addition, although the occupational injury data or reports of relevant institutions provide employers with information to identify risk factors and characteristics related to injury, in practice, further research or intervention areas to prevent occupational injury of electric power workers are also required [9]. However, portable electrical equipment as an electric shock hazard 
medium is seldom examined. Therefore, electrical shock prevention measures for electrical equipment must be identified immediately.

The types of portable electric equipment used in electrical operations are diverse because of the varied demands across electrical operations. Portable electrical equipment typically includes tools carried by workers such as electric hammering machines, electric welding machines, bench grinders, boring machines, and electric hand tools. A study featuring model construction by using Integration DEFinition (IDEF) 0 revealed that construction site safety should be emphasized even before workers enter the construction site. In addition to wearing personal protection equipment (PPE), conducting on-site hazard inspections, confirming that workers have the professional skills needed for the construction operation system, and providing electrical equipment inspection stations, circuits, and extension cords should be implemented to prevent electrocution accidents from defective electrical equipment [10]. However, when workers bring equipment with them into the construction site for respective operations, identifying the qualification of electrical equipment becomes another problem for management.

Therefore, in addition to various inspections of portable electrical equipment, a specific and visual-based mechanism is needed. Visual-based mechanisms must be able to compile various testing data for management personnel to interpret. Therefore, solutions must be identified for compiling various types of testing data and presenting them visually. Visual-based systems should enable management personnel to observe and determine if electrical equipment are qualified and accord with the safety management requirements of the construction site. However, several problems arise from this idea. For example, how can intervention measures eliminate safety concerns associated with defects or the aging of various types of equipment? How should the qualification information of electrical equipment be tested, compiled, and presented visually to lower electrocution risks and strengthen the safety management of construction sites?

In summary, to reduce electrocution hazards from portable electric equipment, this study designed an electrocution prevention early intervention mechanism to lower associated risks and strengthen safety management. The goals of the current study are the following:

- mechanism to lower electrocution risks.

- Establish an identification mechanism for the visual qualification label of portable electrical equipment to strengthen safety management.

\section{Material and methodology}

In this study, narrative text analysis (NTA) was used to identify hazard factors in fatal occupational accidents in which portable electric equipment was the electrocution medium. Subsequently, IDEF3 was used to construct an integrated model to inspect portable electrical equipment (before the portable electrical equipment was brought into the construction site) flowchart of the early intervention mechanism. The researchers hoped that this early intervention mechanism could help lower the risk of electrical shocks in the industry.

\subsection{Compilation and analysis of electrocution hazard factors}

Official accident investigation is useful for analyzing occupational safety. In addition to obtaining information, experiences, and lessons from investigation data for a safe, healthy, and productive workplace, integrating investigation results into a prevention system is more critical [11].

OHS investigation data should be used to analyze hazard factor data from accidents. Only then can effective measures be taken to reduce the frequency of accidents or enhance overall safety standards [12]. For example, analyzed electrocution death cases occurring in the Canadian construction industry from 1997-2007 through text analysis and identified various causation factors such as direct contact with the power supply, low voltage, working outdoors, and other mediums and environmental characteristics [13]. Because accident cases are typically presented as narrative texts, this study employed NTA to explore and unearth information hidden in the texts.

\subsection{Process analysis and model construction}

Model construction projects developed through operation process systems typically focus on structured and automatized promotion. In a study on solar technology, IDEF was used to propose a process model for exploration and identification, and this model accelerated the speed of target confirmation [14]. The model construction problem for process flow could be effectively resolved by using the reusability and extensibility of IDEF0 and IDEF3 to analyze the relationship between the logic and time sequence [15]. During product design, systematizing information through the IDEF model quickly illustrated the relevance between the product's functions, and this was beneficial for establishing a functional platform and information system for enterprise service design. In addition, the model can be used for subsequent research and development [16].

IDEF3 is an IDEF series model construction method used to collect and record process description extraction. Previously, this method was mainly used by institutions. However, this method was gradually employed to improve and develop enterprise operation processes. This method mainly creates units of behavior (UOB) frameworks by using the order relation of events or activities and creates process illustration models based on links, connections, referents, and annotations. When faced with the operation flow of different subjects or regions, simulation modeling of IDEF3 could be used to accelerate the evaluation and optimization of resource use and significantly reduce production and service costs [17]. Therefore, this study use IDEF3 as a communication tool to describe the operation flow of the system. 


\subsection{Electrical safety regulations}

US national codes and standards adopt $\mathrm{mA}$ as the threshold unit for limiting shock currents to users. The standard shock safety threshold value is $50 \mathrm{~V} \mathrm{rms}$. Studies of electrocution cases have indicated that accidents involving rms alternating current (AC) voltage less $50 \mathrm{~V}$ are rare [18]. According to Regulation No. 243 of the Taiwan Occupational Safety and Health Equipment Rules, residual current circuit breakers and shunt circuits should be installed on the connecting circuit of electrical equipment to ensure personnel safety and eliminate possible malfunctions. To prevent electrical shocks, the connecting circuit of moveable or portable electrical equipment should be equipped with a residual current circuit breaker with high sensitivity (current sensitivity rate should be less than $30 \mathrm{~mA}$ ) and high speed (action time less than $0.1 \mathrm{~s}$ ). In addition, according to CNS4782:2017 regulations for voltagereducing devices for $\mathrm{AC}$ arc welding machines, the safety voltage should not be larger than $25 \mathrm{~V}$, and the delay action should be within $1.0+0.3 \mathrm{~s}$. The details are as displayed in Fig. 1:

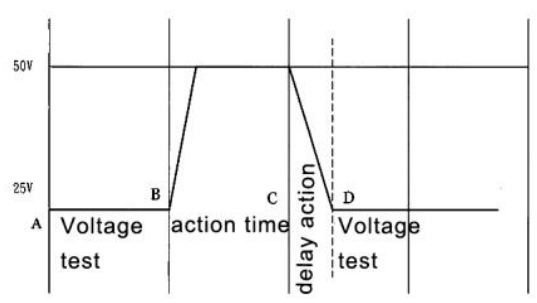

Fig. 1. Voltage flow during testing of electrical welding machine operations $[19,20]$

Under normal circumstances, the rated current capacity of the residual current circuit breaker cannot be smaller than the load current of the circuit. Fig. 2 indicates that when current leakage occurs in the equipment, the leaked current causes the current between the two power cables to become unbalanced (i.e., unequal), which is detected immediately by the zero phase current transformer. When differences between I1 and $\mathrm{I} 2$ exceed the rated current of $30 \mathrm{~mA}$, the residual current circuit breaker activates the switch to cut off the power supply [21].

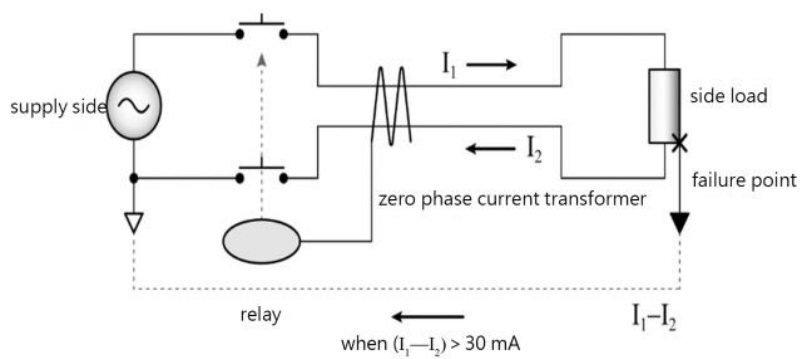

Fig. 2. Current operated residual current circuit breaker [21].

Electrical equipment consists of coils, and their insulation properties may deteriorate after long periods of use. The resistance capacity of the deteriorated insulation layer then lowers accordingly, which causes currents to be leaked into the metal casing. When these leaked currents come into contact with the human body, electrocution hazards occur. The safety and health selfmanagement manual for the construction industry published by the Occupational Safety and Health Administration (OHSA) from Taiwan's Ministry of Labor [22] indicated that before construction machineries such as lifting gears, electrical equipment, working vehicles, moveable scaffolds, and collapsible ladders can enter construction sites, they should be subjected to controlling measures such as ensuring that safety features of the equipment accord with laws and are regulated, conducting of automatic inspections of equipment, and ensuring that equipment parts function properly.

\section{Results and discussion}

\subsection{Establishment of hazard factors}

This study collected and combined the summaries of cases related to electrocution during 2006-2015 among major occupational disasters in the construction industry published by the OHSA. Among reported electrocution cases, 41 featured portable electrical equipment as the electrocution medium. NTA was conducted to identify relevant hazard factors that result in electrocution.

The analysis results indicated 11 types of portable electrical equipment that constitute electrocution mediums: stone crushers, bench grinders, grinding machines, crushers, generators, electric shearing machines, electric screw drivers, electric welding machines, electric hammering machines, electric drilling machines, stirring machines, and boring machines. The hazard factors can be divided into human body hazards (i.e., not wearing PPE); equipment hazards (i.e., lack of grounding, damaged or defective insulation, current leakage, current leakage into the outer casing, lack of barricades, no installation of automatic electrical shock prevention devices, ineffective automatic electrical shock prevention devices, and damaged outer insulation casing of wires); and hazards from power-consuming equipment connected to the circuit of portable electrical equipment (i.e., not installing residual current circuit breakers, having ineffective residual current circuit breakers, and not cutting off the power supply or removing a circuit), as detailed in Table 1 .

From the analysis results, eight human body hazard factors caused electrocution, and all fell under the "not wearing PPE" category. Regarding equipment hazard factors, "damaged or defective insulation" was the category with the highest number of hazard factors (13 hazard factors), followed by "no automatic electrical shock prevention device installed" (seven hazard factors), "no grounding" (six hazard factors), and "current leakage into the outer casing," "ineffective automatic electrical shock prevention devices," and "damaged outer insulation casing of wires" (four hazard factors each). For hazards factors related to power-consuming equipment connected to the circuit of portable electrical equipment, "no residual current circuit breakers installed" was the category with the highest number of 
hazard factors (23 hazard factors). For the number of hazard factors associated with each type of electrical equipment (i.e., electrocution medium), electric welding had the highest number of hazard factors (26 hazard factors), closely followed by electric drilling machines (23 hazard factors).

Table 1. Hazard Factors of Portable Electric Equipment Resulting in Electrical.

\begin{tabular}{|c|c|c|c|c|c|c|c|c|c|c|c|c|c|}
\hline Hazard factor & 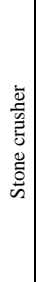 & 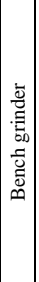 & 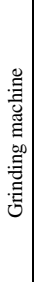 & $\begin{array}{l}\bar{d} \\
\frac{\bar{v}}{2} \\
\bar{U}\end{array}$ & 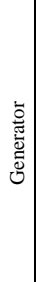 & 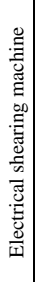 & 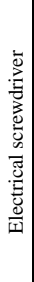 & 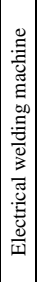 & 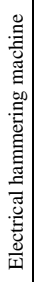 & 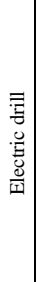 & 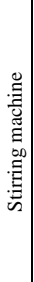 & 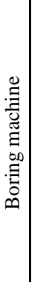 & \\
\hline \multicolumn{14}{|c|}{ Human body hazard } \\
\hline Not wearing PPE & & & & & & & & 5 & 1 & 2 & & & 8 \\
\hline \multicolumn{14}{|c|}{ Equipment hazard } \\
\hline No grounding & & 1 & & & 1 & & & & 1 & 3 & & & 6 \\
\hline Damaged or defective insulation & & 1 & 1 & & & & 1 & 5 & & 5 & & & 13 \\
\hline Current leakage & & 1 & & & & 1 & & & & 1 & & & 3 \\
\hline Current leakage into the outer casing & & & & 1 & & & & & & 1 & 1 & 1 & 4 \\
\hline No barricades & & & 1 & & & & & & & & & & 1 \\
\hline $\begin{array}{l}\text { No automatic electrical shock } \\
\text { prevention devices installed }\end{array}$ & & & & & & & & 7 & & & & & 7 \\
\hline $\begin{array}{l}\text { Ineffective automatic electrical shock } \\
\text { prevention devices }\end{array}$ & & & & & & & & 4 & & & & & 4 \\
\hline $\begin{array}{l}\text { Damaged outer insulation casing of } \\
\text { wires }\end{array}$ & & & & 2 & & & & & & 2 & & & 4 \\
\hline \multicolumn{14}{|c|}{$\begin{array}{l}\text { Hazards from power-consuming equipment connected to the circuit of portable electrical } \\
\text { equipment }\end{array}$} \\
\hline $\begin{array}{l}\text { No residual current circuit breaker } \\
\text { installed }\end{array}$ & 1 & 2 & 1 & 1 & 1 & 1 & 1 & 5 & 1 & 8 & 1 & & 23 \\
\hline $\begin{array}{l}\text { Ineffective residual current circuit } \\
\text { breaker }\end{array}$ & & & & 1 & & & & & & 1 & & & 2 \\
\hline $\begin{array}{l}\text { Power supply not cut off or circuit not } \\
\text { removed }\end{array}$ & 1 & & & & & & & & & & & & 1 \\
\hline Total & 2 & 5 & 3 & 5 & 2 & 2 & 2 & 26 & 3 & 23 & 2 & 1 & 76 \\
\hline
\end{tabular}

The statistics indicated that for the 41 electrocution cases, up to 76 causation factors were involved. When averaged across all cases, each case had an average of 1.85 hazard factors. This clearly indicated that potential risks from portable electrical equipment are not caused by single hazard factors. Therefore, before workers enter a construction site and engage in construction, measures such as properly wearing PPE, examining residual current circuit breakers and circuits from powerconsuming equipment connected to the circuits of portable electrical equipment, and conducting final inspections of portable electrical equipment should be implemented to lower hazard risks.

\subsection{Factors factors omitted in construction safety management}

Strengthening safety inspections for electrical operations in the construction site is not only beneficial for developing a safety climate but also crucial to employers and employees. Regulations on inspection precautions from the Occupational Safety and Health Act state that business entities are responsible for compiling health and safety management responsibilities and informing contractors of such responsibilities before delivering or contracting projects. Business entities are also responsible for urging contractors at all levels to create a workplace that complies with the health and safety conditions specified in the act to reduce the occupational accident rate [23].

The present researchers analyzed the summaries of real electrocution cases and identified six factors that are often omitted in construction safety management: relevant health and safety education and training, automatic inspections, stipulation of health and safety rules for workers, appointment of a labor health and safety management supervisor, establishment or confirmation of coordinating organizations or affairs, and disclosure of required measures against hazard factors (Table 2).

Table 2. Items omitted in construction safety management

\begin{tabular}{lcc}
\hline \multicolumn{1}{c}{$\begin{array}{c}\text { Items omitted in construction safety } \\
\text { management }\end{array}$} & $\begin{array}{c}\text { Number of } \\
\text { cases }\end{array}$ & $\%$ \\
\hline $\begin{array}{l}\text { Relevant health and safety education and } \\
\text { training }\end{array}$ & 34 & $82.93 \%$ \\
$\begin{array}{l}\text { Automatic inspections } \\
\text { Stipulation of health and safety rules for } \\
\text { workers }\end{array}$ & 30 & $73.17 \%$ \\
$\begin{array}{l}\text { Appointment of a labor health and safety } \\
\text { management supervisor }\end{array}$ & 28 & $68.29 \%$ \\
$\begin{array}{l}\text { Establishment or confirmation of coordinating } \\
\text { organizations or affairs }\end{array}$ & 18 & $43.90 \%$ \\
$\begin{array}{l}\text { Disclosure of required measures against hazard } \\
\text { factors }\end{array}$ & 8 & $16.51 \%$ \\
\hline
\end{tabular}

Based on the analysis of the omission rate in 41 electric shock accidents, the results revealed that relevant health and safety education and training was the most omitted $(82.6 \%)$, followed by automatic inspections $(73.17 \%)$, stipulation of health and safety rules for workers $(68.29 \%)$, appointment of a labor health and safety management supervisor (43.90\%), establishment or confirmation of coordinating organizations or affairs $(39.02 \%)$, and disclosure of required measures against hazard factors $(19.51 \%)$.

Among the listed construction safety measures, most must be executed before initiating construction operations. This includes implementing health and safety education and training, distributing relevant work rules, establishing or confirming coordinating organizations or affairs, appointing a labor health and safety management supervisor, and disclosing required measures against hazard factors. All planning must be made in advance and be executed in accordingly. In contrast to other construction safety measures, the execution time and venue for automatic inspections must be confirmed at the construction site. Therefore, autoinspection is a key topic for pre-entry safety examinations [23].

3.3 Compilation and model construction to inspect the early intervention mechanism 
As aforementioned, despite the adoption of safety measures such as using PPE and ensuring the safety of the residual current circuit breakers and circuits of power-consuming equipment on site, the safety of portable electrical equipment carried by the workers is neglected in hazard risk management because of the safety management unit's exclusion of portable electrical equipment from safety management. Therefore, an early intervention mechanism for portable electrical equipment can be employed to lowers this risk.

Human body hazard factors, equipment hazard factors, and hazard factors from power-consuming equipment connected to the circuit of portable electric equipment were used to construct a visual process model through the IDEF3 approach, which was used to construct the portable electric equipment inspection mechanism. Six UOB were used to establish the inspection mechanism: human protection, equipment outer casing inspection, equipment operation inspection, testing the electric welding machine's antishock function, and printing qualification labels. In this mechanism, the workers must first have put on PPE before entering the construction site (UOB1). Subsequently, the static power consumption for portable electric equipment is inspected (UOB2), followed by dynamic inspection of the equipment (UOB3), testing the antishock function of electric welding machines (UOB4), and conducting overcurrent testing for extension cords (UOB5). If the tool is qualified, qualification labels with a fixed validity period are printed (UOB 6) and affixed to the equipment before they can be brought into the construction site. Equipment that failed the inspection are removed from the site. After the equipment is repaired or replaced, it undergoes the same pre-entry testing procedures before being allowed to re-enter the construction site (UOB2UOB6)[19,20]. The inspection process of the early intervention mechanism is illustrated in Fig.3.

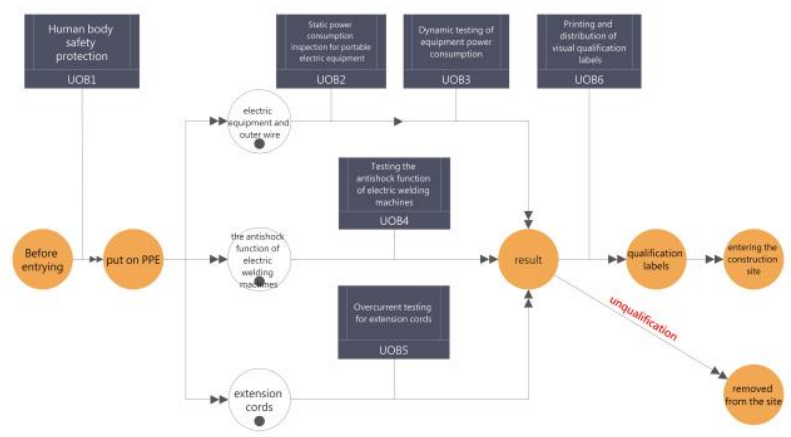

Fig. 3. Inspection process of the early intervention mechanism.

\subsubsection{UOB1: Human body safety protection}

PPE focuses on protective measures for the human body instead of preventive measures. Therefore, when working under conditions with known or potential hazards, workers put on PPE, and its components (e.g., safety helmet, gloves, protective shoes, and googles) can prevent direct contact with hazardous substances [24]. PPE is mainly used to create a barrier between the user and working environment to reduce hazards from being exposed to physical, electrical, thermal, and chemical substances [5]. Therefore, to maintain the most basic level of protection, workers should be given PPE before entering the construction site.

\subsubsection{UOB2: Static power consumption inspection for portable electric equipment}

Before testing, dangerous parts of the equipment are removed (Fig. 4(1)), and the outer casing of the equipment is grounded and fixed to the metal outer casing. Manual and visual inspections are conducted to detect damages in the circuit (Fig. 4(2)). The midsection of the equipment's power lines is placed in water tanks with attached grounding electrodes. The midsection of the equipment's power lines must be completely submerged in the water to be the conductive medium. The supply and plug into the socket of the required voltage should be appropriately connected (Fig. 4(3)). The power supply button is activated but the operation does not commence. After $2 \mathrm{~s}$, the machine changes the power supply automatically to conduct anode-cathode exchange testing. This ensures that the leaked currents are tested using different phase angles. Whether current leakage occurs at the outer casing of the equipment and whether it occurs due to aged or defective outer wire casing are observed, and this is the first stage of static testing. For equipment failures at the first stage of static testing, leaked current values are displayed on the screen. If the leaked current value exceeds the set safe value ${ }^{a}$, the defect indication light turns on and the buzzer emits an alarm (Fig. 4(4)). Only equipment passing the static testing (i.e., no current leakage) can proceed to the second stage of dynamic testing.

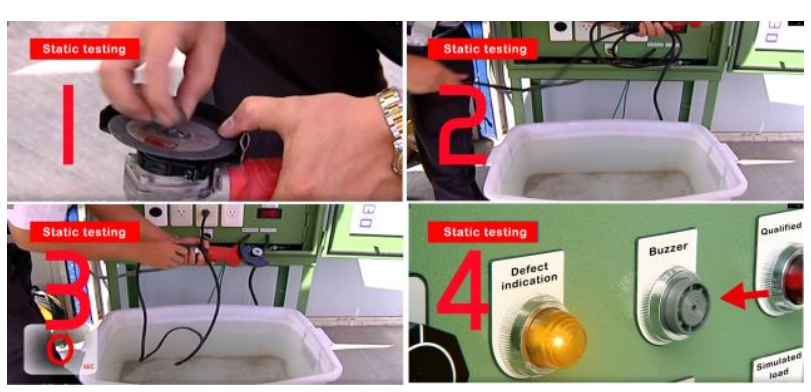

Fig. 4. Static power consumption inspection process for portable electrical equipment.

\subsubsection{UOB3: Dynamic testing of equipment power consumption}

After the equipment passes static testing, it remains in operation for another 4 to $5 \mathrm{~s}$ (Fig. 5(1)). Once the equipment passes testing (i.e., no current leakage), the qualification indication light turns on. The qualification labels can then be printed by pressing the label-printing button (Fig. 5(2)). By contrast, for equipment that failed

\footnotetext{
a The safe leaked current value was determined by referring to the measured "rated sensitivity current" of the zero phase current transformer in the residual current circuit breaker.
} 
testing, the leaked current values are displayed on the screen. If the leaked current value exceeds the set safe value, the defect indication light turns on and the buzzer emits an alarm.

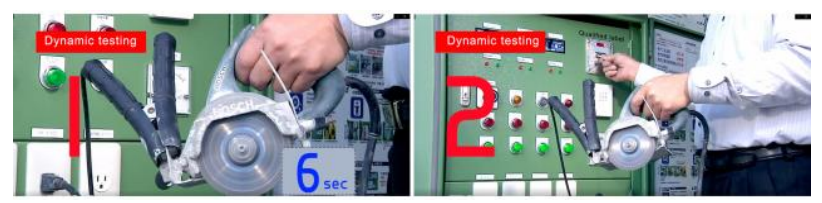

Fig. 5. Dynamic testing process of equipment power consumption.

\subsubsection{UOB4: Testing the antishock function of electric welding machines}

The power supply is connected and plugged into the socket for the required voltage (Fig. 6(1)). The anode and cathode of the electric welding machine are clamped to the anode and cathode of the test clips (Fig. 6(2)). The operation to test the on and off functions of the electric welding machine is activated, and the action time is delayed $^{\mathrm{b}}$. The voltage changes and delayed action time are displayed on the screen (Fig. 6(3)). When the welding machine passes the qualification test, the results are reported orally by the machine, and a qualification label is printed (Fig. 6(4)). In cases where the voltage changes and the delayed action time didoes not match, the results are reported orally by the machine, and no qualification label is printed.

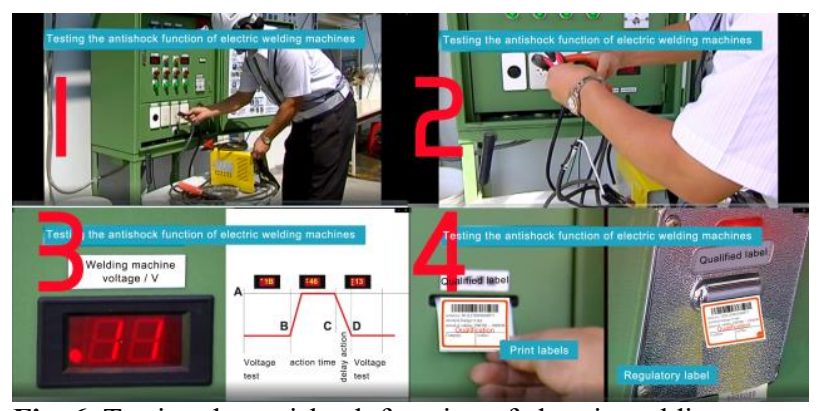

Fig. 6. Testing the antishock function of electric welding machines.

\subsubsection{UOB5: Overcurrent testing for extension cords}

To prevent wire fires, overcurrent testing is conducted for overload protection switches of the extension cords. A low-voltage high-current scheme is used to automatically regulate electrical impedance and adjust the constant current to double the rated current of $15 \mathrm{~A}$ for the product ${ }^{\mathrm{c}}$. Subsequently, any defects that can jeopardize the safety of personnel from the equipment

\footnotetext{
${ }^{\mathrm{b}}$ For the qualification standard, the input voltage was set below $25 \mathrm{~V}$ according to the CNS4782:2017. After the welding was finished, the voltage value reverted back the safe voltage below $25 \mathrm{~V}$ after $1 \mathrm{~s}$ of delay action time $(+0.3)$.

${ }^{\mathrm{c}}$ Tested according to the regulations stipulated in Section 3.1.6 of CNS10917. To speed up testing, a double current of approximately 30 A was used to conduct the high-current withstanding test.
}

are examined. The power supply is connected (Fig. 7(1)) and the simulation load line is plugged into the socket of the extension cord (Fig. 7(2)) to activate the current and fuse tripping function (Fig. 7(3)). A 30-A testing current is delivered, and the extension cord passes the testing if tripping occurs within $20 \mathrm{~s}$ (Fig. 7(4)). If the extension cord passes inspection, the qualification light appears and an approval label is printed. The extension cord is considered defective if tripping does not occur within 20 $\mathrm{s}$, and an alarm is sounded to alert the user conducting the test.

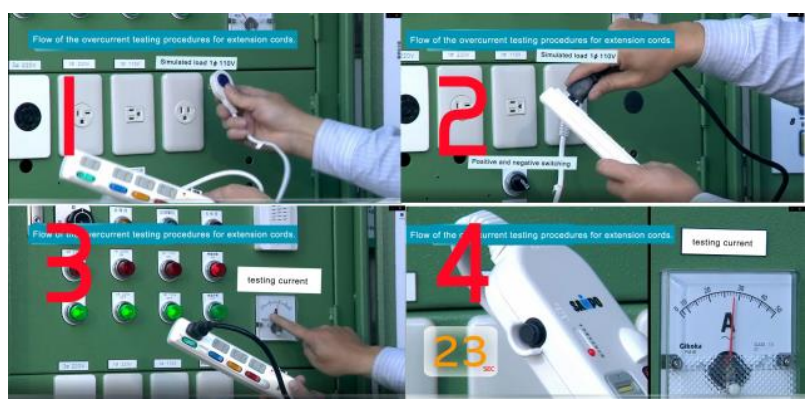

Fig. 7. Flow of the overcurrent testing procedures for extension cords.

\subsubsection{UOB6: Printing and distribution of visual qualification labels}

To prevent the aging of or damage to equipment during usage, the validity period of the qualification labels can be determined according to the needs of management units. For qualification labels with a 3-month validity period, for example, the labels are printed in four colors to differentiate the label stickers for each quarter. The printed items include operation procedural information such the bar code, serial number, validity period, and operation venue (Fig. 8). To strengthen management quality, the label stickers must be cross-cut using an anticounterfeiting knife after the period of validity lapses to prevent repeated usage. Qualified equipment with labels are exempted from pre-entry examination within the validity period.

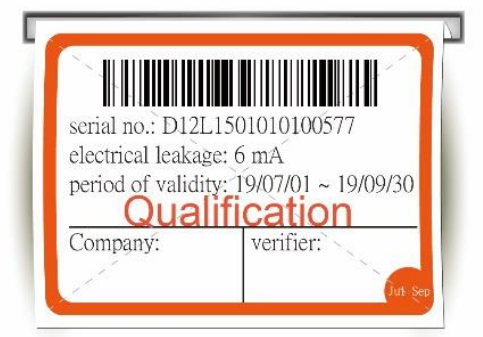

Fig. 8. Visual qualification labels.

\subsection{Inspection electrical equipment before entering the construction site}

Generally, power consumption equipment at construction sites are equipped with a temporary or fixed power supply box. This power supply box must be equipped with a residual current circuit breaker to prevent electrocution accidents from direct or indirect contact of the current with the human body when current 
leakage occurs in the electrical equipment. Therefore, before initiating construction operations, inspections for the power consumption equipment connected to the circuit of the portable electric equipment are conducted. This includes ensuring that a residual current circuit breaker is installed, the residual current circuit breaker works properly, and items such as power supplying cutting and circuit removal work as intended.

\section{Conclusions}

Occupational safety regulations and health laws have been stipulated to prevent electrocution accidents. Temporary power consumption and electrical operations at construction sites should be inspected according to the promulgated regulations. Employers should also establish automatic inspection plans. As proposed by IOSH, Taiwan [21], for every hazard situation that may be encountered by each operation mode in the construction industry, the management unit must take appropriate measures in accordance with the relevant operation regulations. This includes installing a residual current circuit breaker, grounding equipment, using automatic electrical shock prevention devices, using insulating protective equipment, wearing insulating protective equipment, keeping a safe distance, setting up isolation facilities, increasing insulation protection, and taking safety management measures. Therefore, the construction sites should integrate various relevant regulations to form a comprehensive mechanism and implement them.

By adopting the inspection process of the early intervention mechanism, this study enforcing human body protection before allowing construction workers to enter the construction site. Equipment should be subjected to systematic control measures such as static and dynamic testing procedures, testing the antishock function of electric welding machines, and overcurrent testing for the overcurrent protector of extension cords to safeguard labor safety and lower electrocution risks from the equipment. However, visual qualification labels can be used and affixed to electrical equipment to strengthen qualification controls and audit electrical equipment (i.e., judging if electrical equipment is qualified for operations on the construction site) and ultimately enhance the overall electrical operation safety of the construction site. Occupational hazard statistics from the United States and European Union countries have clearly indicated that a healthy and safe work environment is critical for enhancing labor productivity and enterprise competitiveness [25]. For construction units, routine equipment inspection and maintenance can significantly enhance safety and reduce costs from occupational hazards [5]. Therefore, before initiating construction operations at construction sites, shaping a safety climate environment through early interventions and a visualbased mechanism and adopting safety maintenance principles that cover physical, equipment, and environmental factors can help ensure the smooth progression of construction projects.
Small-scale and short-term construction projects may face difficulties implementing the mechanism of this study. Therefore, to achieve the full security of portable electric equipment, the authorities should set up inspection stations in each area to regularly inspect electrical equipment.

\section{Acknowledgments}

This work was supported by the Ministry of Science and Technology of the Republic of China (MOST 107-2221E-035-066).

\section{References}

1. National Safety Council, Injury Facts ${ }^{\circledR}, 2015$ Edition

2. T.A. Fordyce, M.J. Leonhard, H.N. Watson, G. Mezei, X.P. Vergara, L. Krishen. Am. J. Ind. Med., 59, 11, 948-958 (2016).

3. IOSH Taiwan, "Construction industry accidents knowledge platform", Statistics of Major Occupational Disasters (2019), on http://laws.ilosh.gov.tw/ciakp/Statistics1.aspx (accessed 23 October 2019).

4. M.T. Newaz, P. Davis, M. Jefferies, M. Pillay. Saf Sci, 112, 9-17 (2019).

5. Y. Niu, W. Lu, F. Xue, D. Liu, K. Chen, D. Fang, C. Anumba. Saf Sci, 111, 213-223 (2019).

6. X. Yuan, C.J. Anumba, M.K. Parfitt. Autom. Constr., 66, 1-14 (2016).

7. R. Flanagan, C. Jewell, W. Lu, K. Pekericli. AutoID-Bridging the physical and the digital on construction projects. Chartered Institute of Building. ISBN, 1853800191 (2014).

8. W. Wu, A.G. Gibb, Q. Li. Saf Sci, 48, 7, 845-858 (2010).

9. V. Volberg, T. Fordyce, M. Leonhard, G. Mezei, X. Vergara, L. Krishen. J Safety Res., 60, 9-16 (2017).

10. Y.R. Shiau, F.Y. Lo, P.C. Ko. (2020) Manuscript submitted for publication.

11. F. Salguero-Caparros, M. Suárez-Cebador, J.A. Carrillo-Castrillo, J.C. Rubio-Romero. Work, 59, 1, 23-38 (2018).

12. A.A. Raheem, R.R. Issa. Saf Sci, 82, 301-314 (2016).

13. H. Kim, J. Lewko, E. Garritano, B. Sharma, J. Moody, A. Colantonio. Work, 54, 3, 639-646 (2016).

14. H. Baek, B.H. Jun, S.M. Yoon, M. Noh. Journal of Ocean Engineering and Technology, 33, 3, 289-299 (2019).

15. M. Fu, D. Wang, J. Wang, M. Li. IAEAC 2018, 1443-1447, IEEE (2018, October).

16. H.L. Nong, S.P. Li, Y.L. He, Y. Luo. Equipment Manufacturing Technology, 1 (2010).

17. G.N. Khubaev, S.M. Scherbakov, S.N. Shirobokova. European Science Review, 11, 12 (2015). 
18. L.B. Gordon, L. Cartelli and N. Graham. IEEE Trans. Ind. Appl, 54, 6, 6554-6565 (2018).

19. P.C. Ko. patent: CN103537826A (2014)

20. P.C. Ko. patent: TWI496648 (2015)

21. IOSH Taiwan. Handbook for Causes and Prevention of Electrocution in Construction Industry, ILOSH107-T-156, (2018), on https://laws.ilosh.gov.tw/ioshcustom/Web/TechPubl ications/Detail?id=151

22. OHSA Taiwan, Construction industry safety and health self-management operation manual (2014). 2019.11.11 https://www.osha.gov.tw/1106/1196/10141/10151/1 0154/11211/

23. Ministry of Labor R.O.C. (Taiwan). Strengthening the precautions for inspection of Articles 26 and 27 of the Occupational Safety and Health Act (2017) on https://laws.mol.gov.tw/news.aspx?msgid=4577/.

24. J. Hinze, M. Hallowell, K. Baud. J. Constr. Eng. Manag, 139, 10, 04013006 (2013).

25. G.K. Koulinas, P.K. Marhavilas, O.E. Demesouka, A.P. Vavatsikos, D.E. Koulouriotis. Saf Sci, 112, 96-104 (2019). 